\title{
Geochemistry of extrusive rocks at Khun Dan Prakan Chon Dam site, Nakhon Nayok Province, Thailand
}

\author{
Patcharin Kosuwan Jundee ${ }^{\mathrm{a}, *}$, Warisa Hansakulcharoenchai ${ }^{\mathrm{b}}$, Burapha Phajuy ${ }^{\mathrm{a}}$, Piyatida Sangtong ${ }^{\mathrm{b}}$ \\ ${ }^{a}$ Department of Geological Sciences, Faculty of Science, Chiang Mai University, Chiang Mai 50200 \\ Thailand \\ b Geoscience Program, Mahidol University, Kanchanaburi Campus, Kanchanaburi 71150 Thailand \\ *Corresponding author, e-mail: patcharinkosuwan.j@cmu.ac.th
}

Received 10 Nov 2020

Accepted 16 Apr 2021

\begin{abstract}
The extrusive rock samples were collected from outcrops at Khun Dan Prakarn Chon Dam site, Nakhon Nayok Province. They are parts of the Pre-Cretaceous Khao Yai Volcanics located at the southern end of the western LoeiPetchabun-Nakhon Nayok Volcanic Subbelt. Petrological study found that the extrusive rock samples can be classified into four groups: felsic pyroclastic rocks, felsic volcanic lava flows, mafic pyroclastic rocks, and mafic-to-intermediate volcanic/hypabyssal rocks. Geochemical characteristics of the extrusive rock samples, however, can be divided into two groups which are mafic-to-intermediate extrusive rocks and felsic extrusive rocks. Although the major and some trace element signatures of these extrusive rocks are identical to those of typical calc-alkalic magmas, the tectonic discrimination diagrams for least-altered rocks showed that they were different in the magmatic suite and erupting episode; and discriminant diagrams can be successfully used to identify the tectonic environments of extrusive rocks and to evaluate the tectonic setting of a region. Incompatible trace element plot on the tectonic discrimination diagrams for the mafic-to-intermediate extrusive rock samples indicated high affinity with a within-plate tectonic setting, while felsic extrusive rock samples had high affinity with continental volcanic arc tectonic setting. In addition, the tectonic setting of these mafic-to-intermediate extrusive rock samples could be correlated to the Khiao Andesite located in the east of studied area. Dykes could be associated with multiple arc magmatism in Loei Fold Belt and resulted in the subduction in the Late Permian.
\end{abstract}

KEYWORDS: Khao Yai volcanics, Khun Dan Prakan Chon Dam, calc-alkalic rocks, continental arc, within-plate tectonic setting

\section{INTRODUCTION}

The Pre-Cretaceous felsic-to-mafic volcanic/hypabyssal volcanic rocks are of particular interest because they are the evidence to amalgamation of Indochina and Shan-Thai terranes. The PreCretaceous felsic-to-mafic volcanic/hypabyssal rocks in Thailand can be separated into five major belts from west to east: (1) Chiang Rai-Chiang Mai volcanic belt, (2) Chiang Khong-Tak volcanic belt, (3) Nan-Uttaradit volcanic belt, (4) LoeiPhetchabun-Nakhon Nayok volcanic belt, and (5) Sra Kaew-Chanthaburi volcanic belt (Fig. 1a) [1-3]. The studied area is the outcrop in Khun Dan Prakarn Chon Dam site, Mueang Nakhon Nayok District, Nakhon Nayok Province, Thailand that appears in the topographic map at scale of 1:50000, series L7017, sheet 5237 I (Ban Salika), latitudes $14^{\circ} 10^{\prime}-14^{\circ} 30^{\prime} \mathrm{N}$, and longitudes $101^{\circ} 10^{\prime}-101^{\circ} 25^{\prime} \mathrm{E}$ (Fig. 1b) [4]. This area is underlain by Permo-
Triassic volcanic rocks that is part of the volcanic mass known as Khao Yai Volcanics [5]. These volcanic rocks are constituted largely by pyroclastic rocks with minor lava flows and hypabyssal rocks (dikes and plugs) of felsic-to-mafic compositions. The pyroclastic rocks are either fall or flow deposits and are dominated by volcanic breccias and agglomerates. The Khao Yai Volcanics are the southern part of the Loei-Phetchabun-Nakhon Nayok volcanic belt that runs in NNE-SSW direction from Loei through Phetchabun to Nakhon Nayok Provinces [2]. The volcanic rocks in Loei is composed of acidic-to-basic lavas and pyroclastic rocks compositionally equivalent to the lavas with an Ar-Ar age of $237 \pm 12 \mathrm{Ma}$ (Middle Triassic) [6] and, maybe, a southward extension of those in the Phetchabun area. The volcanic rocks in the Phetchabun area have been interpreted as a result of volcanism along an active continental margin [1] in an Early-to-Middle Triassic time, as evidenced by an Ar-Ar dating of amphi- 

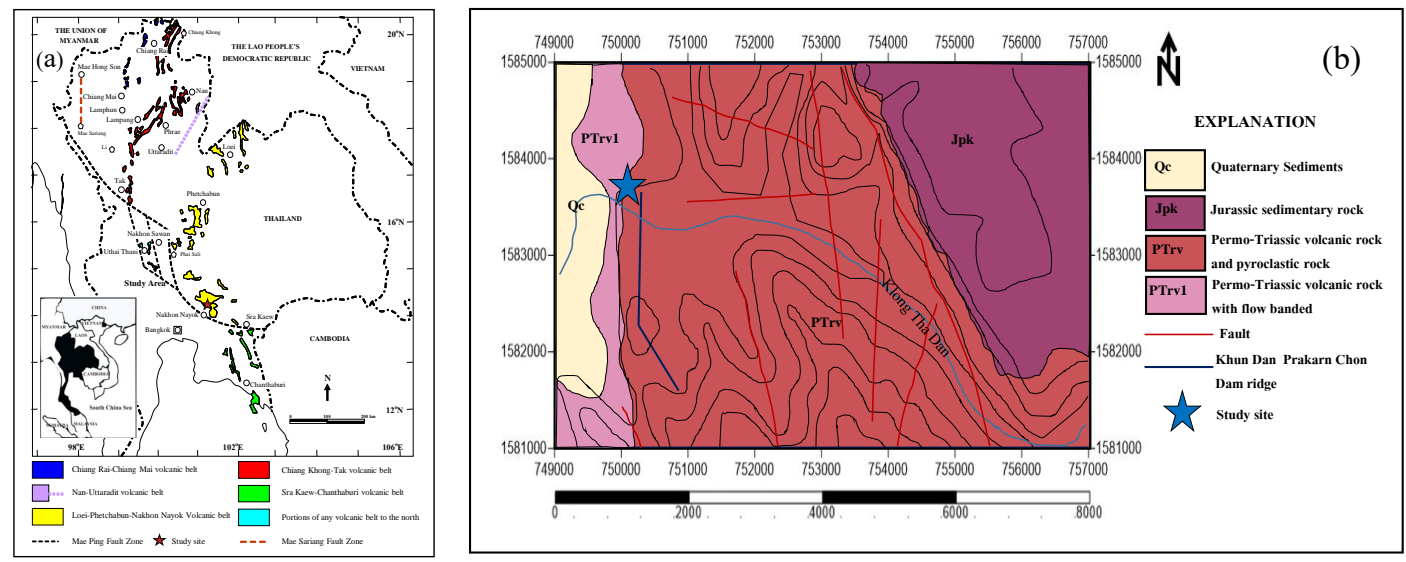

Fig. 1 (a) Distribution of Pre-Cretaceous volcanic rocks in Northern Thailand (b) Geologic map of studied site.

bole which gave a plateau age of $238 \pm 4$ Ma. The volcanic rocks in the Nakhon Nayok area, located in the southern part of the western sub-belt, are also geochemically arc-related magma. The Khao Yai Volcanics ages, obtained from Ar-Ar dating of hypabyssal rocks, and volcanic rocks, located in the northwest of the studied area, are of Jurassic age; hence implying that the dated rocks are younger than the Permian marine sequences $[7,8]$. In addition, the geochemistry of Permo-Triassic Maficto-Intermediate Dyke swarms in the folded layers of the Khao Khwang Fold-Thrust Belt (KKFTB) at the south-western margin of the Indochina Terrane, Central Thailand have ranged from slightly tholeiitic to mostly calc-alkalic. Zircon U-Pb and mica ${ }^{40} \mathrm{Ar} /{ }^{39} \mathrm{Ar}$ ages indicated that the mafic-tointermediate dyke swarms were interpreted as being intruded from the Early Triassic ( $255 \pm 6 \mathrm{Ma}$ ) to the Late Triassic (207 $\pm 2 \mathrm{Ma}$ ). It can be suggested that the rocks were emplaced between the Late Permian and the Late Triassic in a similar orogenic setting and were probably sourced from a more crustally contaminated magma [9]. The volcanic rocks at Wang Nam Khiao Area, Nakhon Ratchasima Province are eastern part of the Khao Yai Volcanics; and are classified as rhyolite, dacite, and andesite whereas dikes are also characterized by andesitic composition. These dikes clearly cut into the volcanic rocks and Late Permian hornblende granite implying that the volcanic rocks and dikes have taken place during/after Late Permian. Whole-rock geochemistry indicated that these suites are related to calc-alkaline hydrous magma, and rare earth element patterns indicated arc magmatism [10]. The aim of the present study was to identify rock types and tectonic settings of eruption of volcanic rock samples.

\section{METHODOLOGY}

\section{Sample preparation}

The 41 least-altered volcanic samples were prepared for whole-rock chemical analysis by splitting into conveniently sized fragments. The fragments were, then, crushed into small chips, using a Rocklabs Hydraulic Splitter/Crusher. Approximately 50-80 g of the chips were cautiously chosen, cleaned, and pulverized for a few minutes by a Rocklabs TungstenCarbide Ring Mill.

\section{Analytical techniques}

Major oxides $\left(\mathrm{SiO}_{2}, \mathrm{TiO}_{2}, \mathrm{Al}_{2} \mathrm{O}_{3}\right.$, total iron as $\mathrm{Fe}_{2} \mathrm{O}_{3}$, $\mathrm{MnO}, \mathrm{MgO}, \mathrm{CaO}, \mathrm{Na}_{2} \mathrm{O}, \mathrm{K}_{2} \mathrm{O}$, and $\mathrm{P}_{2} \mathrm{O}_{5}$ ) were analyzed on fusion discs using a PANalytical Zetium X-ray fluorescence (XRF) spectrometer (wavelength dispersive system), installed at the Kanchanaburi Campus, Mahidol University, Thailand. The standards used were the set of Omnian standards: Omnian-08, -09, -10, -11, -12, -13, -14, -15, -16, and -17 . These chemical species were measured from fusion discs prepared with $0.5 \mathrm{~g}$ powdered sample and $6.5 \mathrm{~g}$ mixer materials consisting of anhydrous lithiumtetraborate $\left(\mathrm{Li}_{2} \mathrm{~B}_{4} \mathrm{O}_{7}\right) 49.75 \%$, lithium metaborate $\left(\mathrm{LiBO}_{2}\right) 49.75 \%$, and lithiumbromide (LiBr) 0.5\%).

Analyses of certain trace elements, i.e. $\mathrm{Ba}$, $\mathrm{Rb}, \mathrm{Sr}, \mathrm{Y}, \mathrm{Zr}, \mathrm{Nb}, \mathrm{Ni}, \mathrm{V}, \mathrm{Sc}, \mathrm{Cr}$, and $\mathrm{Th}$, were carried out using Philips Magix PRO X-ray fluorescence (XRF) spectrometer (wavelength dispersive system). Detection limit for $\mathrm{Ba}$ is $50 \mathrm{ppm}$; whereas for $\mathrm{Rb}, \mathrm{Sr}, \mathrm{Ni}, \mathrm{V}, \mathrm{Cr}$, and $\mathrm{Zr}$ are $10 \mathrm{ppm}$; and $\mathrm{Y}, \mathrm{Nb}$, $\mathrm{Sc}$, and Th are $5 \mathrm{ppm}$. The standards used were 
Table 1 Major oxides and some certain trace elements of the studied volcanic and pyroclastic rocks.

\begin{tabular}{|c|c|c|c|c|c|c|c|c|c|c|}
\hline Sample No. & KD01 & KD02 & KD03 & KD04 & KD05 & KD06 & KD07 & KD08 & KD09 & KD10 \\
\hline \multicolumn{11}{|c|}{ Major oxide (wt $\%$ ) } \\
\hline $\mathrm{SiO}_{2}$ & 60.41 & 57.16 & 64.42 & 63.91 & 74.52 & 60.02 & 63.07 & 76.37 & 74.66 & 71.88 \\
\hline $\mathrm{TiO}_{2}^{2}$ & 1.10 & 1.20 & 0.96 & 0.98 & 0.77 & 0.98 & 0.98 & 0.21 & 0.78 & 0.68 \\
\hline $\mathrm{Al}_{2} \mathrm{O}_{3}$ & 15.87 & 17.77 & 16.79 & 16.72 & 12.89 & 17.38 & 16.95 & 13.73 & 13.57 & 13.83 \\
\hline $\mathrm{Fe}_{2}^{2} \mathrm{O}_{3}^{3}$ & 7.52 & 8.15 & 5.50 & 5.73 & 2.88 & 6.63 & 5.61 & 1.80 & 3.37 & 3.92 \\
\hline $\mathrm{MnO}^{3}$ & 0.15 & 0.10 & 0.06 & 0.07 & 0.03 & 0.11 & 0.07 & 0.03 & 0.04 & 0.04 \\
\hline $\mathrm{MgO}$ & 3.16 & 4.38 & 1.89 & 1.89 & 0.41 & 3.72 & 2.86 & 0.97 & 0.84 & 0.35 \\
\hline $\mathrm{CaO}$ & 4.91 & 3.64 & 1.98 & 2.63 & 0.83 & 4.35 & 3.12 & 2.94 & 1.00 & 1.67 \\
\hline $\mathrm{Na}_{2} \mathrm{O}$ & 5.47 & 5.85 & 6.04 & 5.54 & 3.33 & 4.86 & 5.02 & 0.65 & 3.19 & 7.14 \\
\hline $\mathrm{K}_{2} \mathrm{O}$ & 1.02 & 1.27 & 2.15 & 2.27 & 4.17 & 1.76 & 2.04 & 3.26 & 2.43 & 0.27 \\
\hline $\mathrm{P}_{2}^{2} \mathrm{O}_{5}$ & 0.40 & 0.48 & 0.22 & 0.27 & 0.15 & 0.19 & 0.29 & 0.04 & 0.13 & 0.20 \\
\hline Original Sum. & 99.77 & 99.77 & 99.81 & 99.87 & 99.81 & 99.93 & 99.89 & 99.88 & 99.80 & 99.90 \\
\hline \multicolumn{11}{|c|}{ Trace element (ppm) } \\
\hline $\mathrm{Ni}$ & 89.41 & 86.84 & 13.70 & 16.42 & 9.13 & 81.50 & 78.34 & 14.77 & 16.05 & 14.05 \\
\hline $\mathrm{V}$ & 150.31 & 165.09 & 144.97 & 135.82 & 117.71 & 133.03 & 144.22 & 45.16 & 124.10 & 105.94 \\
\hline $\mathrm{Rb}$ & 36.85 & 29.83 & 51.12 & 57.75 & 98.59 & 45.13 & 87.84 & 74.42 & 64.89 & 10.95 \\
\hline $\mathrm{Y}$ & 23.94 & 23.96 & 26.08 & 26.15 & 30.96 & 24.73 & 26.65 & 25.54 & 29.81 & 25.58 \\
\hline $\mathrm{Nb}$ & 3.99 & 4.17 & 1.33 & 1.70 & 8.04 & 3.50 & 4.27 & 7.37 & 7.80 & 5.60 \\
\hline Th & 0.97 & 0.93 & 4.27 & 4.51 & 13.65 & 2.18 & 1.13 & 10.00 & 11.94 & 5.23 \\
\hline $\mathrm{Sr}$ & 394.98 & 307.86 & 307.43 & 282.96 & 213.85 & 222.29 & 378.45 & 504.36 & 200.50 & 189.42 \\
\hline $\mathrm{Ba}$ & 539.01 & 589.57 & 703.56 & 697.04 & 879.05 & 486.36 & 692.27 & 717.01 & 657.05 & 479.65 \\
\hline $\mathrm{Sc}$ & 17.47 & 11.84 & 7.68 & 10.66 & 5.54 & 14.51 & 11.12 & 11.04 & 3.76 & 7.35 \\
\hline $\mathrm{Zr}$ & 195.03 & 202.25 & 253.47 & 244.57 & 521.37 & 219.30 & 238.52 & 179.28 & 517.40 & 329.38 \\
\hline Sample No. & KD11 & KD12 & KD13 & KD14 & KD15 & KD16 & KD17 & KD18 & KD19 & KD20 \\
\hline \multicolumn{11}{|c|}{ Major oxide (wt $\%$ ) } \\
\hline $\mathrm{SiO}_{2}$ & 63.76 & 77.33 & 54.36 & 54.26 & 56.19 & 55.03 & 56.03 & 55.95 & 58.44 & 73.20 \\
\hline $\mathrm{TiO}_{2}^{2}$ & 1.11 & 0.67 & 1.39 & 1.31 & 1.24 & 1.45 & 1.22 & 1.06 & 1.08 & 0.53 \\
\hline $\mathrm{Al}_{2} \mathrm{O}_{3}$ & 14.49 & 11.71 & 19.35 & 18.88 & 17.97 & 19.31 & 17.63 & 18.67 & 18.91 & 13.32 \\
\hline $\mathrm{Fe}_{2}^{2} \mathrm{O}_{3}$ & 7.42 & 2.61 & 8.78 & 8.88 & 8.67 & 8.56 & 8.36 & 7.92 & 6.96 & 2.19 \\
\hline $\mathrm{MnO}^{\circ}$ & 0.07 & 0.03 & 0.14 & 0.12 & 0.11 & 0.15 & 0.13 & 0.13 & 0.11 & 0.04 \\
\hline $\mathrm{MgO}$ & 0.95 & 0.44 & 3.97 & 4.24 & 4.41 & 4.15 & 5.13 & 3.64 & 3.75 & 0.37 \\
\hline $\mathrm{CaO}$ & 4.94 & 0.96 & 4.33 & 4.99 & 5.27 & 3.64 & 4.81 & 6.23 & 3.33 & 0.95 \\
\hline $\mathrm{Na}_{2} \mathrm{O}$ & 5.04 & 4.02 & 5.34 & 5.32 & 4.76 & 5.96 & 4.14 & 4.63 & 4.66 & 6.59 \\
\hline $\mathrm{K}_{2} \mathrm{O}$ & 1.94 & 2.11 & 1.95 & 1.58 & 1.01 & 1.31 & 2.13 & 1.38 & 2.49 & 2.73 \\
\hline $\mathrm{P}_{2}^{2} \mathrm{O}_{5}$ & 0.29 & 0.13 & 0.40 & 0.42 & 0.37 & 0.44 & 0.44 & 0.39 & 0.27 & 0.08 \\
\hline Original Sum. & 99.81 & 99.83 & 99.87 & 99.85 & 99.83 & 99.88 & 99.73 & 99.64 & 99.77 & 99.56 \\
\hline \multicolumn{11}{|c|}{ Trace element (ppm) } \\
\hline $\mathrm{Ni}$ & 23.40 & 12.44 & 51.05 & 51.26 & 48.83 & 40.21 & 102.64 & 20.59 & 43.06 & 10.63 \\
\hline $\mathrm{V}$ & 156.46 & 114.73 & 198.27 & 178.79 & 192.02 & 188.41 & 171.22 & 171.99 & 155.01 & 89.38 \\
\hline $\mathrm{Rb}$ & 44.10 & 46.92 & 44.65 & 38.09 & 23.76 & 32.56 & 45.75 & 29.62 & 73.79 & 70.31 \\
\hline Y & 23.23 & 28.58 & 23.96 & 24.04 & 23.72 & 23.92 & 24.75 & 23.82 & 25.11 & 30.27 \\
\hline $\mathrm{Nb}$ & 4.28 & 6.70 & 0.67 & 1.28 & 2.38 & 4.83 & 3.01 & 1.70 & 1.39 & 8.03 \\
\hline Th & 3.70 & 15.02 & 5.57 & 1.46 & 4.12 & 1.77 & 1.49 & 1.06 & 4.45 & 14.43 \\
\hline $\mathrm{Sr}$ & 143.29 & 152.69 & 171.05 & 249.77 & 324.48 & 292.95 & 723.40 & 288.86 & 303.35 & 245.66 \\
\hline $\mathrm{Ba}$ & 578.83 & 667.97 & 515.46 & 564.67 & 504.35 & 525.03 & 765.16 & 554.91 & 697.03 & 759.25 \\
\hline $\mathrm{Sc}$ & 16.20 & 3.65 & 14.11 & 15.61 & 14.81 & 10.63 & 16.27 & 19.96 & 14.17 & 4.76 \\
\hline $\mathrm{Zr}$ & 113.20 & 506.75 & 137.86 & 140.84 & 172.48 & 174.29 & 239.15 & 165.79 & 186.96 & 576.13 \\
\hline Sample No. & KD21 & KD22 & KD23 & KD24 & KD25 & KD26 & KD27 & KD28 & KD29 & KD30 \\
\hline \multicolumn{11}{|c|}{ Major oxide (wt\%) } \\
\hline $\mathrm{SiO}_{2}$ & 74.73 & 71.94 & 55.22 & 73.24 & 74.63 & 61.92 & 62.48 & 60.90 & 49.05 & 48.96 \\
\hline $\mathrm{TiO}_{2}^{2}$ & 0.57 & 0.84 & 1.39 & 0.82 & 0.56 & 0.83 & 0.85 & 1.06 & 1.99 & 1.93 \\
\hline $\mathrm{Al}_{2} \mathrm{O}_{3}$ & 13.45 & 14.11 & 18.22 & 13.69 & 13.41 & 17.41 & 17.12 & 16.72 & 18.78 & 19.36 \\
\hline $\mathrm{Fe}_{2} \mathrm{O}_{3}$ & 2.47 & 3.34 & 8.51 & 2.99 & 1.98 & 5.54 & 5.58 & 7.48 & 11.25 & 11.17 \\
\hline $\mathrm{MnO}^{\circ}$ & 0.04 & 0.05 & 0.18 & 0.03 & 0.03 & 0.08 & 0.07 & 0.13 & 0.18 & 0.18 \\
\hline $\mathrm{MgO}$ & 0.35 & 0.58 & 4.09 & 0.46 & 0.27 & 2.75 & 3.06 & 3.68 & 4.94 & 4.92 \\
\hline $\mathrm{CaO}$ & 0.73 & 1.13 & 4.85 & 0.91 & 1.55 & 4.67 & 4.31 & 3.24 & 6.02 & 5.83 \\
\hline $\mathrm{Na}_{2} \mathrm{O}$ & 4.53 & 4.23 & 5.55 & 4.21 & 6.30 & 3.97 & 4.76 & 4.55 & 2.39 & 2.37 \\
\hline $\mathrm{K}_{2} \mathrm{O}$ & 3.06 & 3.67 & 1.54 & 3.52 & 1.21 & 2.61 & 1.55 & 2.11 & 4.79 & 4.70 \\
\hline $\mathrm{P}_{2}^{2} \mathrm{O}_{5}$ & 0.08 & 0.12 & 0.44 & 0.14 & 0.06 & 0.21 & 0.20 & 0.13 & 0.60 & 0.59 \\
\hline Original Sum. & 99.77 & 99.78 & 99.83 & 99.84 & 99.71 & 99.80 & 99.84 & 99.87 & 99.83 & 99.30 \\
\hline \multicolumn{11}{|c|}{ Trace element (ppm) } \\
\hline $\mathrm{Ni}$ & 10.36 & 9.45 & 36.70 & 8.05 & 6.42 & 41.09 & 42.52 & 109.71 & 57.92 & 10.36 \\
\hline V & 91.14 & 121.78 & 189.81 & 111.71 & 82.26 & 121.60 & 133.13 & 141.89 & 250.09 & 91.14 \\
\hline $\mathrm{Rb}$ & 67.92 & 73.23 & 39.96 & 69.49 & 28.57 & 61.66 & 36.00 & 79.34 & 116.59 & 67.92 \\
\hline $\mathrm{Y}$ & 29.88 & 30.29 & 23.99 & 29.55 & 28.26 & 24.50 & 23.67 & 26.21 & 26.96 & 29.88 \\
\hline $\mathrm{Nb}$ & 8.11 & 8.60 & 2.55 & 7.63 & 7.77 & 3.05 & 2.56 & 4.61 & 4.16 & 8.11 \\
\hline Th & 18.99 & 13.15 & 2.23 & 15.32 & 12.00 & 3.52 & 4.03 & 5.03 & 0.75 & 18.99 \\
\hline $\mathrm{Sr}$ & 161.30 & 195.15 & 253.15 & 149.18 & 144.74 & 543.80 & 593.18 & 349.44 & 246.58 & 161.30 \\
\hline $\mathrm{Ba}$ & 725.48 & 785.30 & 504.36 & 840.67 & 557.79 & 711.45 & 615.46 & 731.92 & 444.81 & 725.48 \\
\hline $\mathrm{Sc}$ & 3.30 & 3.90 & 16.76 & 1.77 & 7.06 & 15.04 & 13.11 & 9.94 & 16.66 & 3.30 \\
\hline $\mathrm{Zr}$ & 559.41 & 555.42 & 160.79 & 527.84 & 543.95 & 211.44 & 214.62 & 238.83 & 161.15 & 559.41 \\
\hline
\end{tabular}


Table 1 Continued ...

\begin{tabular}{|c|c|c|c|c|c|c|c|c|c|c|c|}
\hline Sample No. & KD31 & KD32 & KD33 & KD331 & KD34 & KD35 & KD36 & KD37 & KD38 & KD39 & KD40 \\
\hline \multicolumn{12}{|c|}{ Major oxides (wt\%) } \\
\hline $\mathrm{SiO}_{2}$ & 73.62 & 72.85 & 58.83 & 56.79 & 54.40 & 57.74 & 56.46 & 59.99 & 62.94 & 71.02 & 73.87 \\
\hline $\mathrm{TiO}_{2}^{2}$ & 0.37 & 0.39 & 1.15 & 1.20 & 1.36 & 0.95 & 1.24 & 0.91 & 0.84 & 0.57 & 0.60 \\
\hline $\mathrm{Al}_{2} \mathrm{O}_{3}$ & 14.14 & 14.36 & 17.57 & 17.90 & 18.18 & 18.30 & 16.77 & 17.53 & 17.21 & 14.99 & 13.20 \\
\hline $\mathrm{Fe}_{2}^{2} \mathrm{O}_{3}$ & 2.03 & 2.35 & 8.36 & 7.76 & 8.52 & 6.83 & 8.78 & 6.20 & 5.53 & 2.51 & 2.37 \\
\hline $\mathrm{MnO}^{\circ}$ & 0.06 & 0.06 & 0.10 & 0.10 & 0.11 & 0.11 & 0.12 & 0.12 & 0.09 & 0.04 & 0.04 \\
\hline $\mathrm{MgO}$ & 0.69 & 0.77 & 4.50 & 4.38 & 3.79 & 4.04 & 5.15 & 4.02 & 3.23 & 0.95 & 1.21 \\
\hline $\mathrm{CaO}$ & 1.32 & 2.06 & 1.93 & 4.18 & 7.29 & 4.94 & 5.84 & 4.57 & 2.92 & 2.96 & 2.37 \\
\hline $\mathrm{Na}_{2} \mathrm{O}$ & 4.39 & 4.89 & 5.82 & 5.55 & 4.82 & 3.74 & 3.65 & 3.87 & 4.39 & 5.61 & 4.98 \\
\hline $\mathrm{K}_{2} \mathrm{O}$ & 3.30 & 2.20 & 1.28 & 1.64 & 1.13 & 3.13 & 1.56 & 2.57 & 2.64 & 1.26 & 1.31 \\
\hline $\mathrm{P}_{2} \mathrm{O}_{5}$ & 0.08 & 0.06 & 0.46 & 0.51 & 0.41 & 0.22 & 0.43 & 0.22 & 0.21 & 0.08 & 0.07 \\
\hline Original Sum. & 99.85 & 99.89 & 99.86 & 99.77 & 99.80 & 99.68 & 99.77 & 99.74 & 99.75 & 99.84 & 99.72 \\
\hline \multicolumn{12}{|c|}{ Trace elements (ppm) } \\
\hline $\mathrm{Ni}$ & 12.93 & 9.85 & 103.33 & 81.02 & 54.28 & 59.69 & 90.99 & 54.84 & 40.07 & 51.07 & 11.08 \\
\hline $\mathrm{V}$ & 68.25 & 68.10 & 177.83 & 172.04 & 181.83 & 143.05 & 164.00 & 133.46 & 130.10 & 249.79 & 87.64 \\
\hline $\mathrm{Rb}$ & 96.45 & 69.84 & 39.93 & 53.50 & 28.85 & 80.32 & 31.63 & 56.12 & 70.71 & 118.27 & 30.90 \\
\hline $\mathrm{Y}$ & 26.10 & 25.19 & 24.89 & 25.14 & 23.36 & 24.89 & 23.93 & 24.46 & 24.78 & 26.88 & 28.36 \\
\hline $\mathrm{Nb}$ & 3.84 & 4.90 & 2.86 & 4.64 & 2.35 & 2.82 & 3.17 & 3.97 & 2.26 & 3.94 & 6.14 \\
\hline Th & 7.73 & 10.31 & 2.44 & -0.14 & 2.84 & 4.75 & 5.07 & 3.61 & 7.95 & 1.37 & 12.83 \\
\hline $\mathrm{Sr}$ & 363.88 & 416.22 & 274.87 & 271.15 & 299.74 & 665.33 & 746.31 & 687.82 & 739.36 & 236.54 & 240.22 \\
\hline $\mathrm{Ba}$ & 731.56 & 572.16 & 531.16 & 546.67 & 495.93 & 766.68 & 572.17 & 819.22 & 897.48 & 432.94 & 535.95 \\
\hline $\mathrm{Sc}$ & 5.21 & 8.27 & 8.37 & 13.95 & 21.62 & 15.21 & 16.48 & 14.92 & 11.67 & 17.09 & 9.62 \\
\hline $\mathrm{Zr}$ & 239.80 & 243.59 & 199.39 & 198.07 & 142.87 & 201.29 & 235.69 & 225.42 & 229.42 & 158.71 & 535.09 \\
\hline
\end{tabular}

the USGS geochemical reference materials: AGV2, BCR-2, BHVO-2G, BIR-1a, DTS-2b, DNC-1a, W2a, GSP-2, QLO-1a, RGM-2, and STM-2. These chemical species were measured from pressed powder samples prepared with $6 \mathrm{~g}$ sample powder and $0.3 \mathrm{~g}$ XRF MULTI-MIX PXR-200 for trace elements. These procedures were completed and measured at the Department of Geological Sciences, Chiang Mai University.

Major oxides and certain trace elements of the least-altered samples were shown in Table 1. The data for major oxides, in terms of weight $\%$, were used to calculate the Ishikawa alteration index (AI) and the chlorite-carbonate-pyrite index (CCPI) by the following equations:

$$
\begin{aligned}
\mathrm{AI} & =\frac{100\left(\mathrm{MgO}+\mathrm{K}_{2} \mathrm{O}\right)}{\left(\mathrm{FeO}+\mathrm{K}_{2} \mathrm{O}+\mathrm{MgO}+\mathrm{Na}_{2} \mathrm{O}\right)} \\
\mathrm{CCPI} & =\frac{100(\mathrm{FeO}+\mathrm{MgO})}{\left(\mathrm{FeO}+\mathrm{K}_{2} \mathrm{O}+\mathrm{MgO}+\mathrm{Na}_{2} \mathrm{O}\right)}
\end{aligned}
$$

\section{RESULTS AND DISCUSSION}

The results (Table 1) show that the studied samples lie well within limits of the least-altered rocks [11], except KD10 and KD25 which are slightly altered (Fig. 2).

\section{Lithology and petrography}

The volcanic rocks at Khun Dan Prakarn Chon Dam ridge were composed of felsic and mafic volcanic rocks, and equivalent pyroclastic rocks. These rocks occurred as pyroclastic fall and flow deposits,

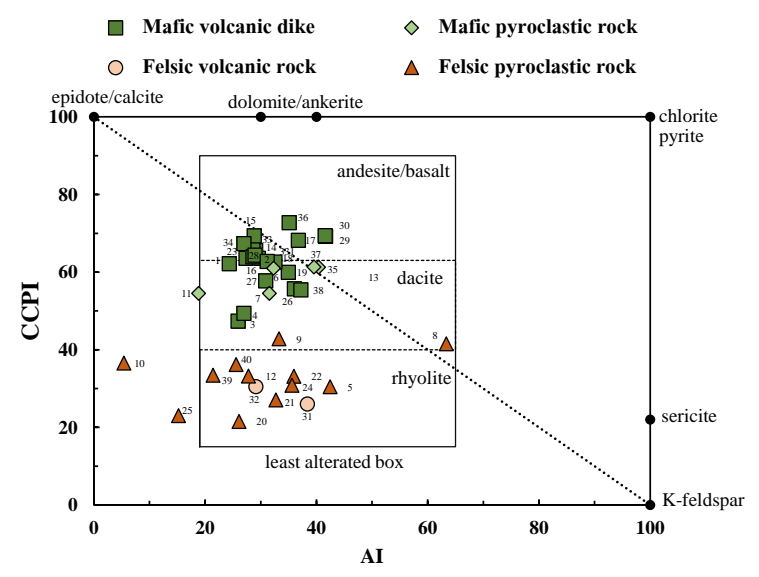

Fig. 2 Alteration Box Plot of rock samples.

lava flows; and those solidified from magma arisen along conduits. Pyroclastic breccias and agglomerates were the most abundant rock types. Fortyone least-altered volcanic rock samples presented in this study were collected from outcrop at the Khun Dan Prakarnchon Dam site, which included felsic volcanic rocks, mafic volcanic rocks (greenish gray, grayish black, and black andesite/basalt), and pyroclastic rock.

The petrographic study can be used to classify these volcanic rocks into 4 groups: felsic volcanic rocks, felsic pyroclastic rocks, mafic volcanic rocks, and mafic pyroclastic rocks (Fig. 3). The felsic volcanic rocks are light brown (KD31 and KD32) rhyolite. They show porphyritic texture with pla- 

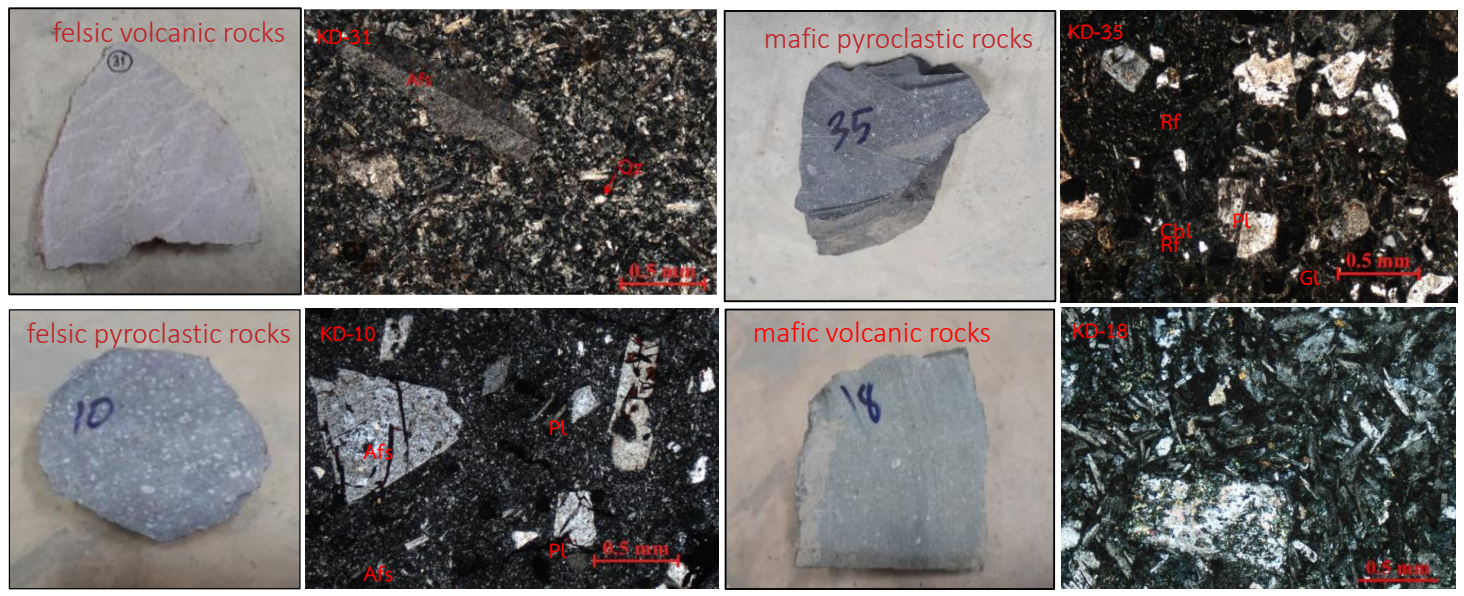

Fig. 3 Lithology and petrography of four groups of rock samples (Afs = alkali feldspar, $\mathrm{Pl}=$ plagioclase, $\mathrm{Qz}=\mathrm{quartz}$, $\mathrm{Ch}=$ chlorite, $\mathrm{Rf}=$ rock fragment, and $\mathrm{Gl}=$ glass $)$.
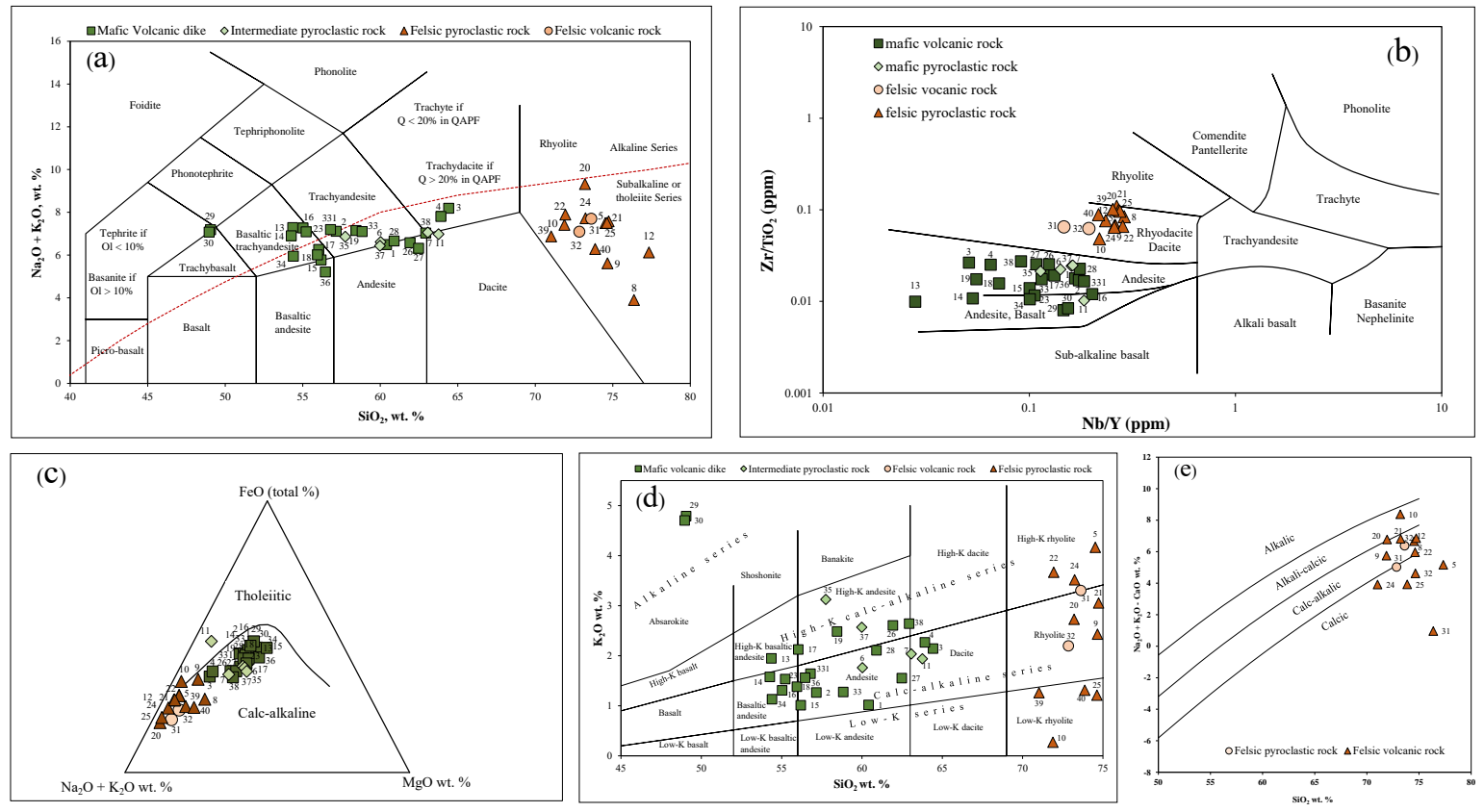

Fig. 4 (a) TAS diagram, (b) $\mathrm{Nb} / \mathrm{Y}-\mathrm{Zr} / \mathrm{TiO}_{2}$, (c) AFM diagram, (d) $\mathrm{SiO}_{2}-\mathrm{K}_{2} \mathrm{O}$, and (e) $\mathrm{SiO}_{2}-\left(\mathrm{Na}_{2} \mathrm{O}+\mathrm{K}_{2} \mathrm{O}-\mathrm{CaO}\right.$ ).

gioclase, alkaline feldspar, and quartz phenocryst$\mathrm{s} /$ microphenocrysts; and groundmass composed of quartz and feldspar. The felsic pyroclastic rocks are grayish red (KD05, KD08, KD09, KD10, KD12, KD20, KD21, KD22, and KD24) and grayish brown (KD25, KD39, and KD40) lapilli tuff and breccia. These felsic pyroclastic rocks show pyroclastic texture that can be classified, based on pyroclasts, to crystal tuff, lapilli tuff, and breccia. The mafic volcanic rocks are dark gray (KD01, KD02, KD03, KD04, KD23, KD26, KD27, KD28, KD29, KD30,
KD33, KD331, KD34, KD36, and KD38) and grayish green (KD13, KD14, KD15, KD16, KD17, KD18, and KD19) andesite/basalt. They show porphyritic texture with plagioclase and pyroxene phenocrysts/microphenocrysts and some samples show sub-ophitic texture. These phenocrysts/mi-crophenocrysts are embedded in the holocrystalline groundmass with a felty or a trachytic texture. The mafic pyroclastic rocks are blackish red (KD06, KD11, and KD35) and blackish gray (KD07 and KD37) lithic tuff. These mafic pyroclastic rocks show pyroclastic texture and 

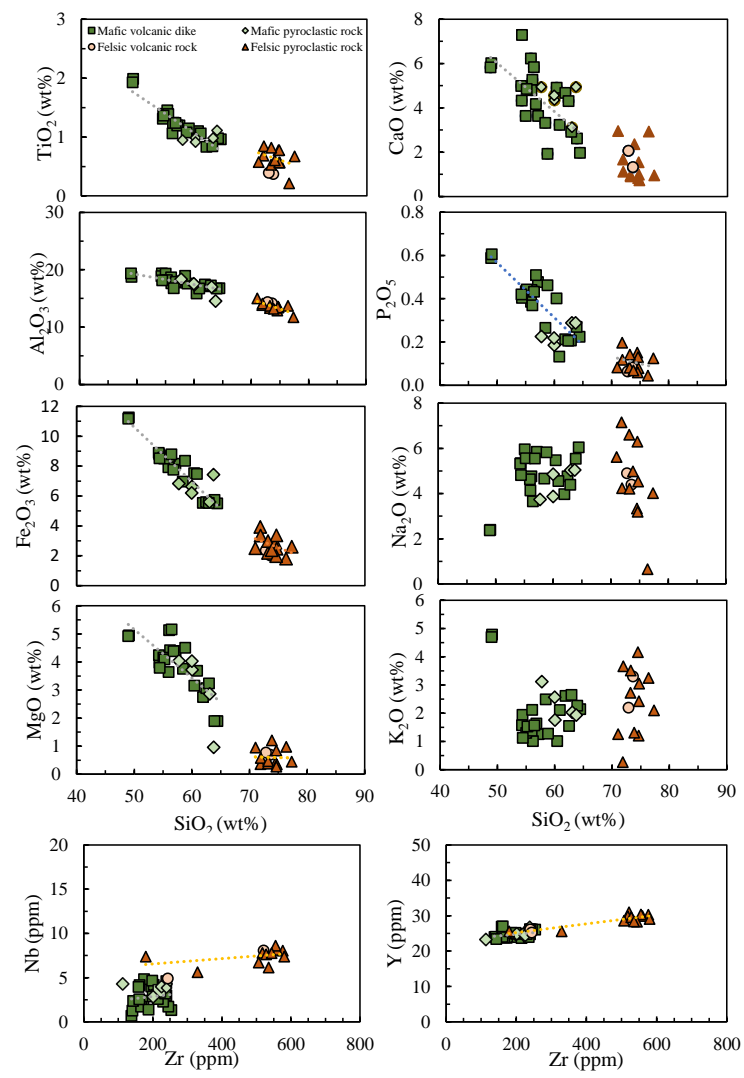

Fig. 5 Harker variation diagrams of $\mathrm{SiO}_{2}$-major oxides; and bivariate diagrams: Zr-Nb and Zr-Y.

can be classified into crystal tuff and lithic tuff.

\section{Magmatic affinity}

Although the studied volcanic rocks were leastaltered, their chemical compositions were unlikely to represent their magmatic source since the rocks underwent varying degrees of alteration and low grade metamorphism. The concentrations of immobile elements could be changed due to either dilution or enrichment of mobile elements. However, the ratios of immobile elements in the primary rocks and the altered rocks remain relatively constant. In this study, relatively immobile elements and relatively immobile element ratios together with some major and minor compositions were used in geochemical interpretation. High field strength elements (HFSE) such as $\mathrm{Ti}, \mathrm{Nb}, \mathrm{Zr}, \mathrm{P}, \mathrm{Rb}$, and $\mathrm{Y}$ were taken in consideration. The data were plotted in several diagrams in order to provide their compositional arrays.

The total alkali silica (TAS) classification diagram [12] (Fig. 4a) was used for nomenclature of rocks and magma by major oxides. The analyzed samples could be divided into 2 groups: (1) maficto-intermediate extrusive rocks with $\mathrm{SiO}_{2}$ content between $48 \mathrm{wt} \%-65 \mathrm{wt} \%$, such as trachybasalt, basaltic trachyandesite, trachyandesite, andesite, and trachyte; and (2) felsic extrusive rocks with $\mathrm{SiO}_{2}$ content between $70 \mathrm{wt} \%-78 \mathrm{wt} \%$, such as rhyolite. Based on immobile elements, the $\mathrm{Nb} / \mathrm{Y}$ $\mathrm{Zr} / \mathrm{TiO}_{2}$ diagram [13] (Fig. 4b) could be classified into mafic extrusive rocks group with the $\mathrm{Zr} / \mathrm{TiO}_{2}$ and the $\mathrm{Nb} / \mathrm{Y}$ ranging from 0.008 to 0.027 and 0.028 to 0.202 , respectively. The compositional field of andesite and a few andesite/basalt and the felsic extrusive rocks had $\mathrm{Zr} / \mathrm{TiO}_{2}$ and $\mathrm{Nb} / \mathrm{Y}$ ranging from 0.048 to 0.109 and 0.147 to 0.289 , respectively. The values were in the compositional field of rhyodacite and a few rhyolites. Most samples were in subalkaline series. In addition, the relationships of $\mathrm{Na}_{2} \mathrm{O}+\mathrm{K}_{2} \mathrm{O}, \mathrm{FeO}$, and $\mathrm{MgO}$ on the alkali, $\mathrm{Fe}$, and $\mathrm{Mg}$ oxides (AFM) diagram [14] (Fig. 4c), $\mathrm{K}_{2} \mathrm{O}$ and $\mathrm{SiO}_{2}$ [15] (Fig. 4d), and $\mathrm{Na}_{2} \mathrm{O}+\mathrm{K}_{2} \mathrm{O}-\mathrm{CaO}$ and $\mathrm{SiO}_{2}$ [16] (Fig. 4e) suggested that most extrusive rocks are calc-alkaline to high-K calc-alkaline series, except a few felsic pyroclastic rocks are low-K (tholeiite) series.

The $\mathrm{SiO}_{2}$ variation diagrams for major oxides and bivariate diagrams of $\mathrm{Nb}-\mathrm{Zr}$ and $\mathrm{Y}-\mathrm{Zr}$ (Fig. 5) showed that the values for $\mathrm{FeO}, \mathrm{TiO}_{2}, \mathrm{MgO}, \mathrm{CaO}$, and $\mathrm{P}_{2} \mathrm{O}_{5}$ of the studied rocks slightly decreased, while values for $\mathrm{Al}_{2} \mathrm{O}_{3}, \mathrm{~K}_{2} \mathrm{O}$, and $\mathrm{Na}_{2} \mathrm{O}$ slightly increased throughout the fractionation. Although the relationship between incompatible-element pairs for the least-altered rocks such as $\mathrm{Nb}-\mathrm{Zr}$ and $\mathrm{Y}$ $\mathrm{Zr}$ were positively linear, the trends of felsic and mafic rocks were not continuous, which signified that they were not co-magmatic suite. They might have formed by different degrees of partial melting of a common source rock or by different degrees of fractional crystallization of the same parental magma. The former was unlikely as the patterns of the incompatible-element pairs did not trace back to zero intercept.

\section{Tectonic setting}

Several tectonic discrimination diagrams were used to deduce tectonic settings. Some major and trace elements discrimination diagrams could be used to suggest the former tectonic environment of a suite of magma.

For the studied mafic extrusive rocks, they appeared to be calc-alkali basalt on Ti-Zr-Y diagram (Fig. 6a) and Ti-Zr-Sr diagram (Fig. 6b) [17]. The Ti-Zr-Y-Nb variation diagram was used for discrimi- 
(a)
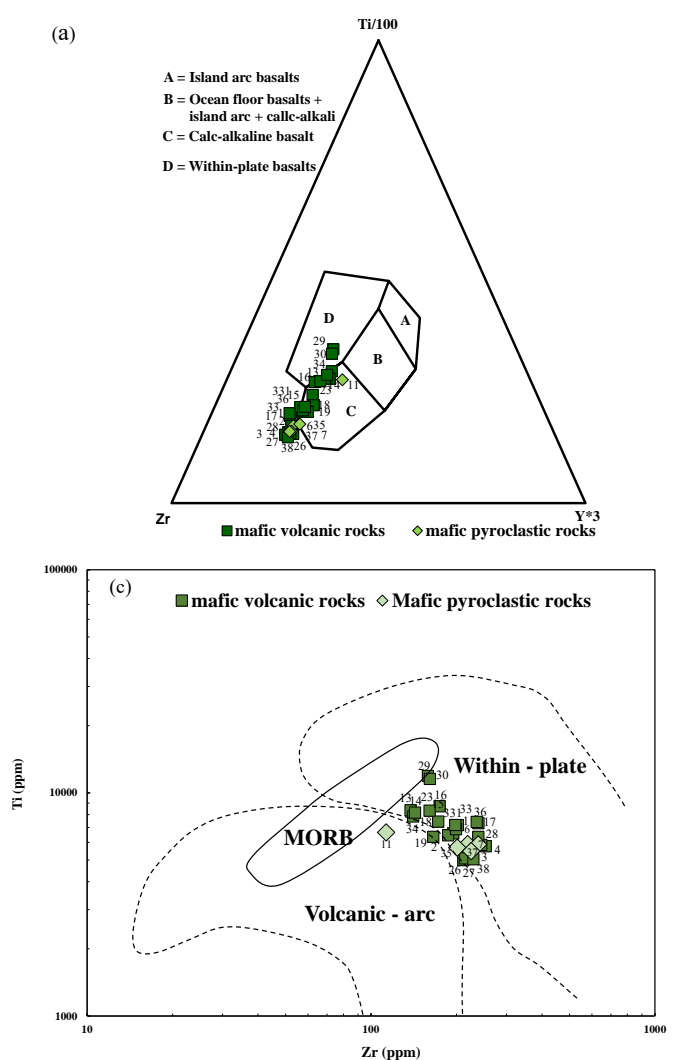

(b)
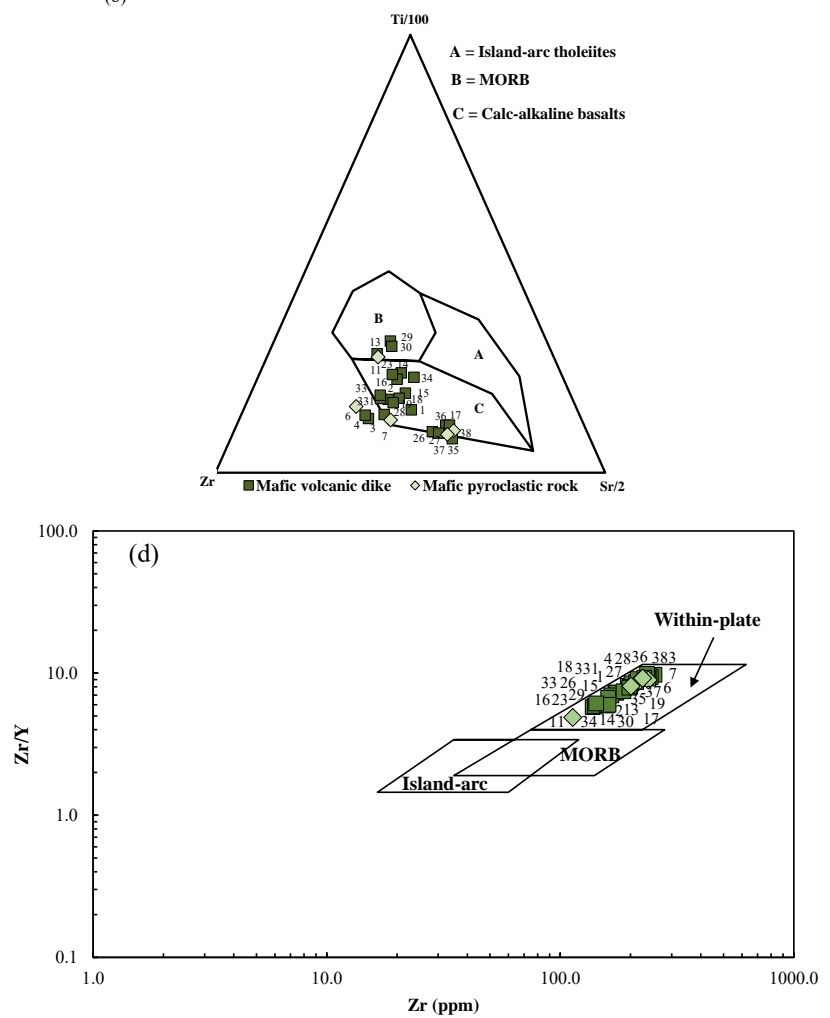

Fig. 6 Tectonic discrimination diagrams of studied mafic volcanic rocks: (a) Zr-Ti-Y, (b) Zr-Ti-Sr (c) Zr-Ti, and (d) Zr$\mathrm{Zr} / \mathrm{Y}$.
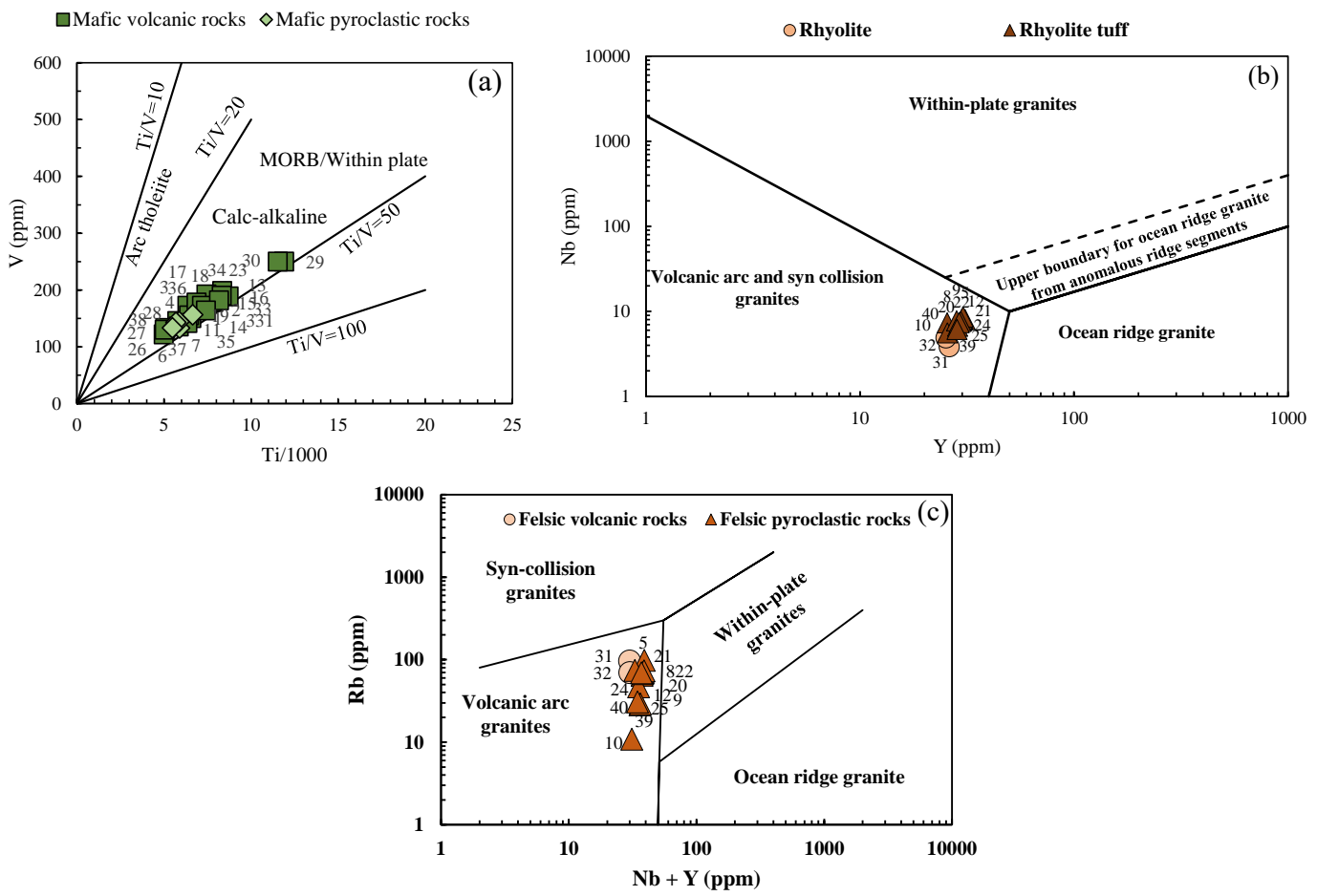

Fig. 7 Tectonic discrimination diagrams of studied extrusive rocks: (a) Ti-V, (b) Y-Nb, and (c) Nb+Y-Rb. 
nating the tectonic settings of studied mafic volcanic rocks. Their tectonic settings were within-plate tectonic setting basalts and supported by their positions on the plots of Zr-Ti [18] (Fig. 6c) and Zr-Zr/Y [19] (Fig. 6d). However, all mafic extrusive rock samples were calc-alkaline basalts on Ti-V diagram [20] (Fig. 7a).

The felsic extrusive rocks were plot on the Nb$\mathrm{Y}-\mathrm{Rb}$ variation diagram, which was used in tectonic discrimination of felsic extrusive rocks. Their tectonic settings were the volcanic arc and syn-collision granites supported by their positions on the plots of Y-Nb (Fig. 7b) and Nb+Y-Rb (Fig. 7c) [21].

Although the rock types from petrographic studied of the extrusive rock samples were varied, their geochemical characteristics were different in magma suite and tectonic settings. According to petrographic study, the rocks could be divided into four sub-groups based on texture and mineral composition; while the geochemically extrusive rock samples could be divided into two sub-groups based on $\mathrm{SiO}_{2}$. The mafic-to-intermediate rock samples shared the distributions of elements such as HFSE $(\mathrm{Nb}, \mathrm{Ti}, \mathrm{P}), \mathrm{Th}, \mathrm{Zr}$, and $\mathrm{Y}$ indicating high affinity with a within plate tectonic setting, while the felsic rock samples were continental volcanic arc tectonic setting. In addition, the Khao Yai Volcanics at Khun Dan Prakarnchon Dam were correlated with the Wang Nam Khiao Volcanics [10], as rhyolite, dacite, and andesite were calc-alkaline magma resulted from continental arc subduction. Moreover, the andesitic dikes were within-plate environments taken place during/after volcanic eruption. It implied that they were associated with multiple arc magmatism in Loei Fold Belt and resulted in the subduction in the Late Carboniferous-Early Permian to Late Permian.

\section{CONCLUSION}

The rock outcrops at Khun Dan Prakarn Chon Dam site were Pre-Cretaceous extrusive rocks (Khao Yai Volcanics). The petrographic study results could be used to classify the rock samples into four groups; felsic pyroclastic rocks, felsic volcanic rocks, mafic pyroclastic rocks, and mafic volcanic/hypabyssal rocks. The felsic volcanic rocks, which are lava flows, showed slightly porphyritic textures; and the felsic pyroclastic rocks showed pyroclastic texture with lapilli to block fragments. The intermediate to mafic volcanic/hypabyssal rocks showed slightly to highly porphyritic textures with sub-ophitic texture might indicate dikes and plugs. The mafic pyroclastic rocks showed pyroclastic texture with ash to lapilli pyroclastic fragments. The geochemically extrusive rocks were classified into two groups: (1) intermediate to mafic extrusive rocks (felsic pyroclastic and felsic volcanic rocks) as subalkalic andesite and subalkalic andesite/basalt and (2) felsic extrusive rocks (mafic pyroclastic and mafic volcanic/hypabyssal rocks) as subalkalic rhyodacite and subalkalic rhyolite. Although major and certain trace elements suggested that the volcanic rocks were mostly calc-alkalic rocks, they originated from different magmatic suite erupted in different episodes. The results of this study well support the model of multiple arc magmatism in LoeiPhetchabun-Nakhon Nayok Volcanic Belt.

Acknowledgements: The authors would like to acknowledge the financial supports from the Faculty of Science, Chiang Mai University and the Igneous Rocks and Related Ore Deposits Research Laboratory (IROL), Department of Geological Sciences, Faculty of Science, Chiang Mai University.

\section{REFERENCES}

1. Boonsoong A, Panjasawatwong Y, Metparsopsan K (2011) Petrochemistry and tectonic setting of mafic volcanic rocks in the Chon Daen-Wang Pong area, Phetchabun, Thailand. J Island Arc 20, 107-124.

2. Jundee PK, Limtrakun P, Boonsoong A, Panjasawatwong Y (2017) Petrography and REE geochemical characteristics of felsic to mafic volcanic/hypabyssal rock in Nakhon Sawan and Uthai Thani Provinces, Central Thailand. Chiang Mai J Sci 4, 1722-1734.

3. Phajuy B, Singtuen V (2019) Petrochemical characteristics of Tak volcanic rocks, Thailand: Implication for tectonic significance. ScienceAsia 45, 350-360.

4. Jungyusuk N, Khositanont S (1992) Volcanic rocks and associated mineralization in Thailand. In: Proceedings of the National Conference on Geologic Resources of Thailand-Potential for Future Development, Bangkok, Thailand, pp 522-538.

5. Department of Mineral Resources (2014) Geological Map of Nakhon Nayok, Department of Minerals and Resources, Bangkok, Thailand.

6. Intasopa S, Dunn T (1994) Petrology and Sr-Nd isotopic systems of the basalts and rhyolites, Loei, Thailand. J Southeast Asian Earth Sci 9, 167-180.

7. Chutakositkanon V, Charusiri P, Sashida K (2000) Lithostratigraphy of Permian marine sequences, Khao Pun Area, Central Thailand: Paleoenvironments and tectonic history. J Island Arc 9, 173-186.

8. Chutakositkanon V, Hisada K, Charusiri P, Arai S (2001) Tectonic significance of detrital chromian spinels in the Permian Nam Duk Formation, central Thailand Geosci $J$ 5, 89-96.

9. Arboit F, Collins AS, Morley CK, Jourdan F, King R, 
Foden J, Amrouch K (2016) Geochronological and geochemical studies of mafic and intermediate dykes from the Khao Khwang Fold-Thrust Belt: Implications for petrogenesis and tectonic evolution. Gondwana Res 36, 124-141.

10. Hunyek V, Sutthirat C, Fanka A (2020) Magma genesis and arc evolution at the Indochina terrane subduction: Petrological and geochemical constraints from the volcanic rocks in Wang Nam Khiao area, Nakhon Ratchasima, Thailand. J Front Earth Sci 8, ID 271.

11. Large RR, Gemmell JB, Paulick H, Huston DL (2001) The alteration box plot: A simple approach to understanding the relationship between alteration mineralogy and lithogeochemistry associated with volcanic-hosted massive sulfide deposits. Econ Geol 96, 957-971.

12. Le Bas MJ, Le Maitre RW, Streckeisen A, Zanettin B (1986) A chemical classification of volcanic rocks based on the total alkali-silica diagram. J Petrol 27, 745-750.

13. Winchester JA, Floyd PA (1977) Geochemical discrimination of different magma series and their differentiation products using immobile elements. $J$ Chem Geol 20, 325-343.

14. Irvine TN, Baragar WRA (1971) A guide to the chemical classification of the common volcanic rocks. Can
J Earth Sci 8, 523-548.

15. Ewart A (1982) The mineralogy and petrology of Tertiary-Recent orogenic volcanic rocks: With a special reference to the andesitic-basaltic compositional range. In: Thorpe RS (Ed) Andesites: Orogenic Andesites and Related Rocks, Wiley, Chichester, pp 25-95.

16. Frost BR, Barnes CG, Collins WJ, Arculus RJ, Ellis DJ, Frost CD (2001) A geochemical classification for granitic rocks. J Petrol 42, 2033-2048.

17. Pearce JA, Cann JR (1973) Tectonic setting of basic volcanic rocks determined using trace element analyses. Earth Planet Sci Lett 19, 290-300.

18. Pearce JA, Alabaster T, Scheton AW, Searle MP (1981) The Oman ophiolite as a cretaceous arc-basin complex: Evidence and implications. Philos Trans $R$ Soc A 300, 299-317.

19. Pearce JA, Norry MJ (1979) Petrogenetic implications of $\mathrm{Ti}, \mathrm{Zr}, \mathrm{Y}$, and $\mathrm{Nb}$ variations in volcanic rocks. Contrib Mineral Petrol 69, 33-47.

20. Shervais JW (1982) Ti-V plots and the petrogenesis of modern and ophiolitic lavas. Earth Planet Sci Lett 59, 101-118.

21. Pearce JA, Harris NBW, Tinder AG (1984) Trace element discrimination diagrams for the tectonic interpretation of granitic rocks. J Petrol 25, 956-983. 